\title{
Narcotici Anonimi Una risorsa per la società
}

Questo articolo, gentilmente favorito dal prof. Alfio Lucchini (Past President di FeDerSerD e Direttore Scientifico di questa rivista), nasce dalla sensazione che il mondo degli Operatori nelle Dipendenze possa essere meglio informato sulla nostra Associazione, talvolta confusa con centri di trattamento privati (anche molto costosi), tal'altra assimilata a una forma di setta religiosa chiusa e/o autofilantropica.

NA nasce invece dal movimento di Alcolisti Anonimi sul finire degli anni Quaranta.

Il censimento del 2015 dà una stima di più di 67.000 riunioni settimanali in 139 paesi, ed evidenzia una forte diffusione anche in Medio Oriente, in particolar modo in Iran, dove si contano più di 350.000 membri.

L'approccio di Narcotici Anonimi al recupero dalla tossicodipendenza è completamente non professionale e si basa sull'auto e mutuo aiuto.

Noi crediamo che il programma di NA funzioni così efficacemente soprattutto per il valore terapeutico che si ha quando un dipendente aiuta un suo simile.

L'impegno di Narcotici Anonimi per cooperare con la società può essere meglio compreso nel contesto dei nostri Dodici Passi e Dodici Tradizioni.

Il nostro Dodicesimo Passo per il recupero individuale incoraggia ogni singolo membro a provare a "portare il messaggio" ai dipendenti.

Tra le nostre Dodici Tradizioni ci sono alcuni principi che ispirano il nostro approccio, sia come gruppi locali sia come organizzazione, verso le altre componenti della società:

- La nostra organizzazione ha lo scopo di far sapere che si può smettere di usare, perdere il desiderio di farlo e trovare un nuovo stile di vita.

In altri termini, che forse possiamo aiutare a vivere senza droghe, a recuperare dagli effetti della dipendenza e a raggiungere uno stile di vita stabile e produttivo.

- Le nostre pubbliche relazioni cercano, anche attraverso un dialogo con gli attori sociali preposti (professionisti, forze dell'ordine, mezzi di comunicazione di massa), di raggiungere i dipendenti verso Narcotici Anonimi senza essere apertamente o eccessivamente promozionali.

- La nostra associazione è aperta a chiunque voglia smettere di usare droghe, indipendentemente dalla particolare sostanza usata e senza preclusioni di età, razza, identità sessuale, convinzioni, religione o assenza di religione.

- Nelle nostre relazioni con le altre organizzazioni manteniamo una politica di "cooperazione senza affiliazione".

Questo approccio ci permette di lavorare con altre realtà sociali senza distrarci dal nostro scopo primario.
Ciò significa che:

1. non diamo supporti e/o avalli, né ci opponiamo ad altre organizzazioni o ad altri approcci terapeutici, per problemi correlati alla dipendenza;

2. non permettiamo ad altre organizzazioni di usare il nome di Narcotici Anonimi per i loro programmi;

3. non finanziamo altre organizzazioni, né accettiamo finanziamenti provenienti dall'esterno della nostra organizzazione;

4. non prendiamo posizione su questioni pubbliche, neanche su quelle relative alla dipendenza (es. riduzione del danno e simili).

NA non è un'organizzazione antidroga né proibizionista e i suoi membri e servitori non prendono posizione riguardo alla depenalizzazione o alla legalizzazione della sostanze stupefacenti. In pratica, non siamo favorevoli né contrari ai programmi per lo scambio di aghi e siringhe, alle terapie sostitutive o alle politiche per la riduzione del danno.

Noi lavoriamo con chiunque voglia offrire il nostro aiuto/supporto/sostegno ai propri pazienti, senza interferire con i regimi terapeutici o nelle relazioni medico-paziente.

Noi incoraggiamo chiunque intenda collaborare con Narcotici Anonimi ad acquisire familiarità con i Dodici Passi e le Dodici Tradizioni attraverso il nostro libro "Funziona: come e perché", disponibile presso il nostro Comitato per le pubbliche Relazioni. I gruppi attivi da qualche tempo di solito pubblicano I'elenco delle loro riunioni, indicando giorno, ora e luogo dove si tengono gli incontri e talvolta dando altre informazioni sullo specifico formato delle riunioni.

Potete ottenere uno di questi elenchi, di solito nella forma più aggiornata, visitando www.na-italia.org.

Ci sono due specie di riunioni di Narcotici Anonimi.

Chiunque può partecipare a una riunione "aperta" per vedere di persona di cosa si tratta.

Le riunioni "chiuse" sono invece riservate solo ai membri.

Prima di recarvi a una riunione accertatevi presso il servizio telefonico o tramite l'Elenco Gruppi se quella a cui volete partecipare sia "chiusa" o "aperta".

Inoltre, i comitati locali di Pubbliche Relazioni possono condurre su richiesta delle presentazioni presso le organizzazioni sociali, gli operatori dei servizi per il trattamento, gli staff clinici, i responsabili delle politiche di intervento e i ricercatori.

Per concludere, NA si propone dunque come un'ulteriore risorsa per la società, gratuita, assolutamente libera ed aperta a qualsiasi forma di collaborazione con le strutture esistenti.

Per qualsiasi ulteriore informazione, vi invitiamo a visitare il nostro sito www.na-italia.org o a contattarci al nostro numero telefonico nazionale 3394116259.

Vi ringraziamo per l'attenzione. 